Amazonía Peruana, Volumen XVII, Nº 34, 2021; pp. 113-125

\title{
CREACIÓN
}

\section{MUESTRA POÉTICA}

\author{
Dina Ananco \\ dinananco@gmail.com \\ (D) https://orcid.org/0000-0002-1844-6575
}




\section{Yamaiya pamuk}

Yamaiya pamukcha

Urukamtai itipchincha itipruiñatsu

Nuwa ememau tui tuiñakua

Mukchirin tawasan ekenas wajaiñawai

Uun inkua aujam aya iima wajaiñawai

Yaunchukia pamukka anchauwa armiayi

Nanki takakuapi

Itipkan itipraku

Wari, chichamtin, tseken wajau armiayi 


\section{Los pamuk de hoy}

Los pamuk de hoy

Por qué no se ponen el itip

Las mujeres son creídas de tanto decir

Están paradas con la corona apenas puesta en la cabecita

Cuando un uun les saluda, solo observan

Los pamuk tradicionales no eran así

Con la lanza en la mano

Con itip puesto

Fuerte, conversador, tseken se presentaban 


\section{Tseartik}

Tseartik asanku

Aya yumameakaj

Ukura takusna

Anku weakai

Perekasa umarna

Ukur ukur mejenkan

Yumameakaj

Urukamtain juniaj

Tusanu, entsa iyankan

Michamchat jawakasa main

Waka ekemsanku

Yumameakaj

Nujintan yuachaitkun

Wiki yumameajai

Mikutjai metektsu

Jinkara aeptusrum

Mantuatarumtai

Yumamtsukka pujumain nekapeatsjai

Tseartikaitkuncha

Aya najaanaran

Nanakin wemain nekapeajai 


\section{Tseartik}

Como soy Tseartik

Solo me como a mí misma

Con la yuca brava en la mano

$\mathrm{Al}$ atardecer

Tomando de un bocado

Perfumada de ukur

Me como a mí misma

¿Por qué me pasa esto?

Diciendo, me sumerjo al agua

Y me refresco jawakasa

Vuelvo y me siento

Y me como a mí misma

No porque haya comido el huevo

Yo sola me como

Igual que Mikut

Seguro teniéndome amarrada

Me matarán

No puedo vivir sin comerme a mí misma

Cual Tseartik

Transformándome

Siento que me iré volando 


\section{Ajaim}

Ajaimpisha urukuchik aya

Ejemachu atatkusha

Urukamtain Etsa nukurincha yuruaya

Usukiar pujujakmaunmasha

Ajaimpisha wariñak usukijakuit

Pujutincha urukamtain nekajakchauwait

Etsayash ima senchi ajakuit

Etsa maachmauwaitkusha urukmainkit

Ajaimpika iwanchiash aya

Tikich iwanchiash

Anentai kasamin arma nu iwanchiash 


\section{Ajaim}

Cómo habrá sido Ajaim

Para no saciar su hambre

Por qué habría comido a la madre de Etsa

Si se vivía con el poder de los conjuros

¿Qué maldecía Ajaim?

¿Por qué no predecía su futuro?

¿Etsa tenía más poder?

¿Qué sería de él si no lo hubiese asesinado Etsa?

¿Ajaim era diablo?

¿Otro tipo de diablo?

¿Los que roban el corazón? 


\section{Iwa}

Wika, shuaraitak yukarta nunaka Iwa, tajai

Yaunchuksha nita amutmajakaruiti

Tinkishap, tiship tura tsere atsakainka

Wampistika amukmau amain

Yamai urukawa

Iiña amutmaktaj tuiñawai

Amuki weajistai

Yamai jata iñarmatai

Namak timu ajunkam urukawa

Numantin pata jaiñawai

Tatartuiñawai, jukataiñawai,

Nmakaitkusha chukuat chukuat jaiñawai

Nuna ya puyattruktata

Utir, shikmi ajapar pujustatji

Iwaka mutsuke yuruamsha

Tuin puja, nui pujus wishikramas iirmatsuash

Jiñakrin 


\section{Iwa}

Le llamo Irwa a los que siendo humanos nos consumen

Antiguamente nos exterminaban

Si no fuera por tinkishap, tiship o tsere

Los wampis habríamos sido extinguidos

Como ahora

Que buscan exterminarnos

Seguro vamos en ese camino

Ahora con la pandemia

Como cuando le echas barbasco a los peces

Así se enferman los familiares

Tatar tatar hacen bulla, roncan

Chukuat chukuat están cual peces

¿Quién se preocupa por ello?

Lloraremos y continuaremos viviendo

Iwa, aunque le hayan comido su cerebro

En donde esté, seguramente nos mira sonriendo

Cuando nos morimos 


\section{Panki nuwaitjai}

Panki tiranki asan

Panki kakarma imanaitjai

Wi miñakainka

Nunka kenertiñaiti

Waimakusha

Miña kara achitrukchau asa

Yaja wajas iiru wajauwaiti

Panki nuwa asan

Init enentaimran

Yukartumain nekapnitjai

Kakakan emajtuiñakai

Tirankur uunmaiñak

Chichaman antuiñachkai

Kajejamain nekapeajai

Tsunki nuwa asan 


\section{Mujer boa soy}

Como nieta de la boa

Soy tan fuerte como la boa

Cuando yo me aproximo

Tiembla la tierra

Los waimaku también

Porque no me han quitado mi fuerza

Observándome están desde lejos

Como soy mujer boa

Reflexionando profundamente

Siento deseos de comer a alguien

Cuando me tratan mal

Cuando mis nietos que crecen

No me obedecen

Tengo deseos de enojarme

Por que soy mujer sirena 


\section{Atsut}

Atsut nuwa asan

Yakiya

Yuranmin jeena puju

Yuranmin nukuri

Yuranmia iman

Yurankim uruk iirsawak nuna kuitamin

Shuara wakani wean naka nakaku kintamin

Shuara wakanijai puju nuwa

Iyakrisha ejemainchau

Yuranmijai metek, jearmainchau 


\section{Atsut}

Mujer Atsut soy

Del cielo

De la casa de neblina

Y la madre

Semejante a la neblina

Que cuida la neblina, su mirada

Que aguarda el alma del fallecido durante el día

Mujer que vive con el alma del humano

Imposible de verla por completo

Inalcanzable como la neblina 